\title{
Improving Visual Field Awareness among Collegiate Softball Athletes: A Randomized Trial
}

\author{
Diana R. Feldhacker ${ }^{1}$, Whitney Lucas Molitor ${ }^{2}$ \\ ${ }^{1}$ School of Pharmacy and Health Professions, Creighton University, Omaha, NE 68178, USA; \\ ${ }^{2}$ School of Health Sciences, University of South Dakota, Vermillion, SD 57069, USA \\ Received 2019-08-26; accepted 2019-12-05 \\ DOI : $10.3724 /$ SP.J.1329.2020.01007
}

开放科学 (资源服务)标识码 (OSID):

\begin{abstract}
Objective: The purpose of this study is to determine the efficacy of high-performance vision training on enhancing visual field awareness among collegiate softball athletes, as well as to compare effectiveness of results based on intervention type which consisted of Dynavision ${ }^{\mathrm{TM}} \mathrm{D} 2$ or therapist-led non-machine vision training. Methods: Twenty-one athletes were randomized to two groups: Group A participated in Dynavision ${ }^{\mathrm{TM}}$ D2 training, and Group B participated in non-machine, therapist-led visual training. Each group engaged in vision training, 15 minutes, twice weekly for six weeks, following a pre-established vision training protocol. After pretesting, a 2-week familiarization period allowed both groups to orient to the Dynavision ${ }^{\mathrm{TM}} \mathrm{D} 2$ training device. Group-specific interventions included dynamic skill training framed in a cognitive and sensory approach. A Repeated Measures Analysis of Variance (ANOVA) was used to analyze changes in means across multiple observations (pretest, posttest, and follow-up testing) within- and between-subject factors (Groups A and B) to determine differences among Dynavision ${ }^{\mathrm{TM}} \mathrm{D} 2$ quadrant and ring hits and speeds of reaction time. Parametric testing was used as data followed a normal distribution. Statistical significance was set at $\alpha \leqslant 0.05$. Results: The study demonstrated that high-efficacy vision training was effective in improving the peripheral field awareness of collegiate softball athletes. This was demonstrated via mean hits and speeds within quadrants and rings of the Dynavision. Both Group A and B demonstrated statistically significant improvements in number of hits within quadrants 1,2 , and 4 as well as rings 3, 4, and 5. In addition, both groups demonstrated statistically significant improvements in speed within all quadrants and all rings. Therefore, both Dynavision ${ }^{\mathrm{TM}} \mathrm{D} 2$ training and alternative vision training may be effective training methods to improve the visual field awareness and reaction time of an elite athletic population; however, the Dynavision ${ }^{\mathrm{TM}} \mathrm{D} 2$ may be a more effective tool. There was a statistically significant difference between Group A and Group B, with Group A experiencing more significant improvements than Group B in number of hits in quadrants 2 and 4 and rings 2 and 3 as well as speeds within quadrants 1 and 2 and ring 2 and 5. Follow-up testing, completed in the absence of additional intervention, confirmed study results and skill retention at 1and 2-month follow-ups. Conclusion: Peripheral vision contributes to sport performance, with implications on an athlete's reaction time, anticipation, and overall safety while on the field. Research indicates improvement of visual field awareness can be gained through use of the Dynavision ${ }^{\mathrm{TM}} \mathrm{D} 2$, yet these studies primarily involve subjects with established field loss or post injury, rather than as a training method among well athletes. The results from this study demonstrate the adaptability of the visual system for those with normal field of vision.
\end{abstract}

KEY WORDS Dynavision ${ }^{\mathrm{TM}}$ D2; vision training; occupational therapy; softball; athletes; visual field; peripheral vision

The visual system is a critical component of sport performance. Ball-game sports, such as softball, incorporate multiple visual demands, especially of the visual field. Softball athletes must utilize visual fields to effectively participate in batting and fielding components of the game ${ }^{[1-2]}$. Specifically, athletes require central and peripheral visual field integration to determine, in fractions of a second, appropriate actions and reactions; the ability to do this accurately and efficiently determines success within the sport.

Central vision is comprised of details within the immediate viewing environment, while peripheral vision includes indirect visual information outside the central field of view ${ }^{[1-3]}$. Peripheral visual fields provide information related to space, orientation, and movement; within softball this includes multiple environmental

引用格式:FELDHACKER D R,MOUTOR W L. 改善大学垒球运动员视野意识的随机试验研究 [J]. 康复学报,2020,30(1):26-33.

FELDHACKER D R, MOLITOR W L. Improving visual field awareness among collegiate softball athletes: a randomized trial [J]. Rehabilitation Medicine, $2020,30(1): 26-33$

DOI: $10.3724 /$ SP.J.1329.2020.01007 
components, such as the ball, teammates, and opponents ${ }^{[3]}$. Adequate peripheral vision is vital for anticipation, reaction time, and the overall safety of athletes during sport ${ }^{[34]}$. Inefficiency, inattention, or reduction in peripheral visual fields necessitate head turning, slowing reaction time, increasing risk of injury, and decreasing adaptability and success within the sport ${ }^{[1,3]}$. Therefore, peripheral vision is an essential component and predictor of an athlete's performance in sport.

Despite the important role vision plays in sport performance, vision training, let alone specific peripheral field training, is often not a factor of emphasis within traditional sport performance training. Few studies have explored vision training among collegiate athletes, and even fewer have related to peripheral awareness. One study compared visual training methods for peripheral vision, depth perception, and visual reaction time among athletes ${ }^{[5]}$. Results indicated that peripheral vision training was the most effective approach for improving multiple visual skills. Peripheral training resulted in cross over improvements to both depth perception and reaction time. Addressing peripheral vision in training has the potential to translate to improvements in other skills, making it a key component that influences sport performance of athletes ${ }^{[5]}$. Schwab and Memmert (2012) ${ }^{[3]}$ also studied peripheral visual training among youth athletes and reported positive findings regarding the ability to improve peripheral perception through visual intervention. Madsen and Blair (2017) ${ }^{[6]}$ incorporated peripheral vision training into their protocol, however, did not report findings related specifically to peripheral awareness.

The traditional approach to improving peripheral awareness for sport performance is through vision training, typically initiated by athletic trainers, optometrists, or other professionals specializing in sports, vision, and sport performance ${ }^{[7]}$. The best approach to vision training has yet to be established, and there is inconsistent and lacking support for efficacy of vision training programs. Even fewer studies exist regarding vision training specific to visual field awareness, and none specific to softball athletes. One study by Appelbaum, Lu, Khanna, \& Detwiler (2016) ${ }^{[8]}$ utilized a vision training approach focusing on visual clarity, contrast sensitivity, visual perception, near-far quickness, peripheral awareness, eye-hand coordination, and response time. Investigators found that vision training did improve peripheral awareness.

A second study by Clark and colleagues (2015) ${ }^{[9]}$ combined traditional vision training with machine-led training with use of the Dynavision. The Dynavision ${ }^{\mathrm{TM}}$ D2 is a computerized light board containing 64 lights displayed within five concentric rings and separated into four quadrants (further described below). This instrument is used to examine and train the visual field among a variety of populations, including collegiate athletes ${ }^{[10]}$. The Dynavision ${ }^{\mathrm{TM}} \mathrm{D} 2$ has been shown reliable in improving awareness of and reaction to central and peripheral stimuli ${ }^{[11-12]}$. Four different modes: A, B, $\mathrm{C}$, and D (described below) are incorporated for both training and testing. The board may be manipulated to illuminate in its entirety, within quadrants, or by rings to train visual field awareness and reaction time. Corresponding results report a number of hits, accuracy, and speed in each ring and quadrant, according to specific mode settings. Clark and colleagues (2015) ${ }^{[9]}$ found that ocular motor and visual conditioning with use of the Dynavision ${ }^{\mathrm{TM}} \mathrm{D} 2$ and additional vision training methods positively impacted hand-eye coordination, improving the accuracy and control of the intraocular and extraocular muscles of the eye and the muscle memory of the arms and hands. These improvements indicated an ability to maintain central focus while successfully receiving and reacting to information from the peripheral visual fields. These visual enhancements increased athletes' on-field awareness, reducing concussive injuries by approximately $84 \%{ }^{[9]}$.

Despite these positive findings, limited research specific to use of the Dynavision ${ }^{\mathrm{MM}} \mathrm{D} 2$ for visual field training with athletes exists. Yet, within the application to other populations, the effectiveness of use for enhancing peripheral awareness is well documented. For example, Dynavision ${ }^{\mathrm{TM}} \mathrm{D} 2$ training has been found to significantly improve the visual field awareness of clients who have experienced acquired brain injuries and older adults ${ }^{[13-15]}$. As a means of documenting similar findings among athletes, the purpose of this study was to determine the efficacy of high-performance vision training on enhancing visual field awareness among collegiate softball athletes, as well as to compare effectiveness of results based on intervention type which consisted of Dynavision ${ }^{\mathrm{TM}} \mathrm{D} 2$ or therapist-led non-machine vision training.

\section{Methods}

\subsection{Research design}

This study was completed using experimental-type research with a two-arm, randomized controlled trial, mixed within-group and between-group design. Prior to recruitment an a-priori power analysis was performed, indicating a minimum of 10 participants would be necessary to reach $80 \%$ power to detect a medium effect size using Cohen's criteria of alpha $=0.05$ for statistical significance. Participants $(n=21)$ were randomly allocated, using a randomized computer generator, into one of two intervention groups: Group A or Group B. Both groups followed the protocol established by Feldhacker and Lucas Molitor (2019) ${ }^{[16-17]}$. Group A $(n=10)$ engaged in intervention using the Dynavision ${ }^{\mathrm{TM}}$ D2, and Group B ( $n=11)$ completed intervention us- 
ing therapist-led vision training exercises which mimic the skills required for use of the Dynavision ${ }^{\mathrm{TM}} \mathrm{D} 2$, especially visual and motor reaction time, peripheral field awareness, and central-peripheral integration. Investigators and participants were not blind to group allocation.

\subsection{Research participants}

Participants were recruited via convenience sampling from a women's Division I softball program 1ocated at a university in the Midwestern United States through contact with the team's coach. Inclusion criteria required that participants be active collegiate softball players from the university who were between the ages of 18 and 22. Twenty-two healthy athletes with no current or history of visual conditions agreed to participate and were randomly allocated into one of two intervention groups. Demographic information was collected from each participant, including age, academic grade rank (freshman-senior), and number of years playing softball. All participants were assigned a random, numerical identifier to anonymize data that were collected and prevent biases during data analysis. During the study, one of the athletes withdrew from the softball team, and her data were not included in the analysis. Data were collected at the team's training facility located on campus. See figure 1 for associated consort diagram.

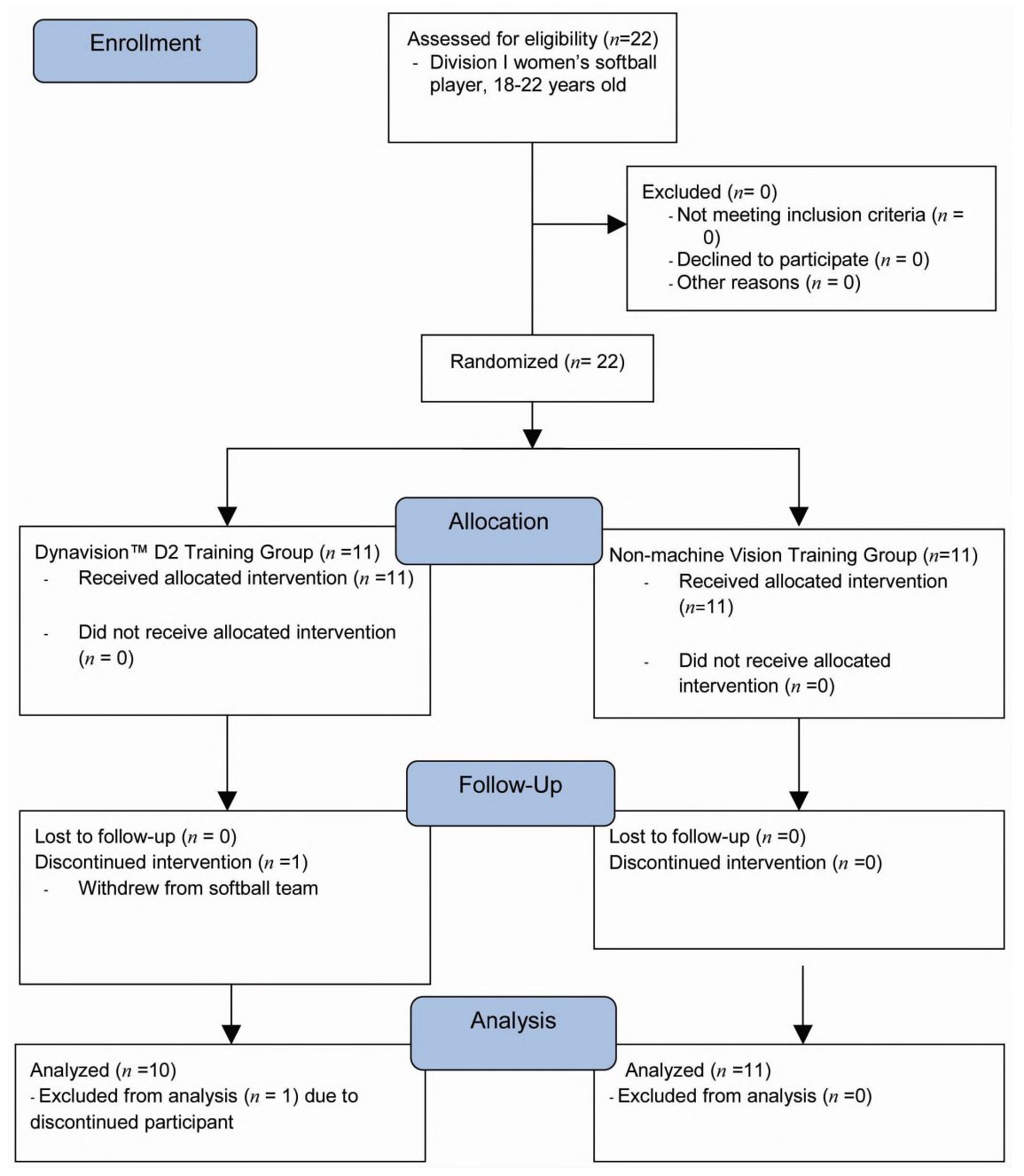

Figure 1 Consort Flow Diagram

1.3 Research instruments

1.3.1 Vision testing Visual assessments were performed at baseline and post-testing to determine eye dominance and visual abilities in the areas of confrontation, smooth pursuits, field of view, convergence, saccades, accommodation, and static and dynamic visual acuity.
1.3.2 Dynavision $^{\mathrm{TM}} \mathrm{D} 2$ The Dynavision $^{\mathrm{TM}} \mathrm{D} 2$ has four modes: A, B, C, and D. Mode A is a proactive mode; during use, a single red light illuminates and remains lit until hit. Once hit, another single red light illuminates in a random location on the board. This continues for the preset duration of the mode. In accordance with Feldhacker and Lucas Molitor (2019) ${ }^{[16-17]}$, Mode 
A was used both for assessment and intervention. The Dynavision $^{\mathrm{TM}}$ D2 has been found to be a reliable method to assess reaction time (ICC2, 1:0.73-0.80) among an athlete population 121 . Data collected from Mode A were analyzed per ring and quadrant hits and speed in order to assess peripheral visual field awareness. See figure 2 for visual depiction of this apparatus.
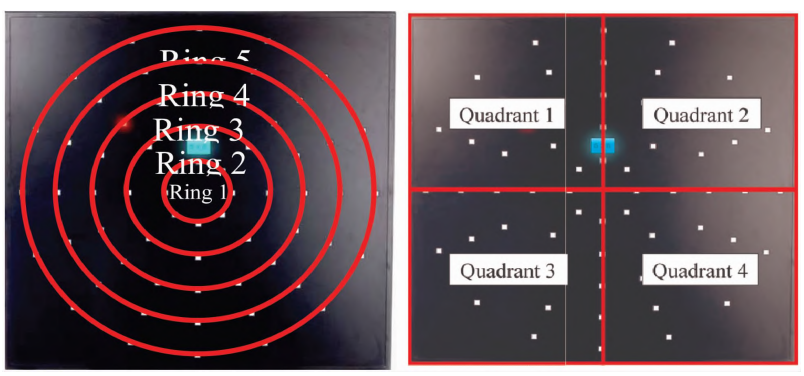

Figure 2 Ring and quadrant allocation on Dynavision $^{\mathrm{TM}} \mathrm{D} 2$ apparatus

The 6-week intervention consisted of determining baseline reaction time and increasing the cognitive, vestibular, and sensory load while concurrently reducing the time each light was illuminated, as a means of training reaction time. During the intervention, results from Mode A were utilized to individually set reaction time for Mode B, unique to training with this protocol ${ }^{116-17]}$. Mode B differs from Mode A in that red lights remain illuminated for the preset length of time before moving to and illuminating in a different, random location, even if unhit. Mode B was utilized in training to increase the demand placed upon the athletes through required integration of central focus, peripheral awareness, and visual anticipation, providing an individualized and appropriate challenge to promote the development and improvement of skills.

Mode $\mathrm{C}$ was utilized during intervention to improve and strengthen peripheral visual field awareness, pursuits, scanning, and attention ${ }^{|16-17|}$. Lights illuminated in the outermost ring and traveled in clockwise and counterclockwise directions at a set speed. The athletes were instructed to follow the path of lights with their eyes while their heads remained in a stationary position; during this, investigators monitored oculomotor movements and visual tracking. Mode D is an assessment mode which was not utilized during this study. This mode consists of six tests which are used to calculate an average reaction time, but it does not provide information specifically related to peripheral awareness.

1.3.3 Therapist-led vision training Vision training strategies were implemented to replicate skills addressed with use of the Dynavision: Saccades, pursuits, reaction time, accommodation, central-peripheral integration, attention, and peripheral awareness.
Feldhacker and colleagues (2019) ${ }^{|16-17|}$ conducted a study to determine efficacy of vision training on improving reaction time of collegiate softball athletes. The same protocol was utilized for Group B in this study. See table 1.

Table 1 6-week Intervention Protocol for Group B

\begin{tabular}{cl} 
Time & Therapist-led vision training \\
\hline Week 1 & Exercises: Saccades, pursuits, and reaction time \\
Week 2 & Exercises: Accommodation and central-peripheral \\
& integration \\
Week 3 & Exercises: Attention and reaction time \\
Week 4 & Exercises: Saccades, pursuits, and central-peripheral \\
& integration \\
Week 5 & Exercises: Accommodation and reaction time \\
Week 6 & Exercises: Attention and central-peripheral integra- \\
& tion
\end{tabular}

\subsection{Procedure}

Approval from the University's Institutional Review Board (IRB) was obtained prior to the study. After receiving approval, athletes were recruited via an email sent by the first author. All athletes who met inclusion criteria provided informed consent prior to the start of the study. Data collected during the study were deidentified and stored in a locked cabinet. A manual of the protocol ${ }^{|16-17|}$ was created which contained verbal directions, session tasks, and data recording sheets. From this manual, five graduate-level occupational therapy students were trained by principal investigators (study authors). Interventions were rehearsed among investigators prior to each session to ensure consistency and reliability with data collection procedures. Competence was checked and ensured by principal investigators prior to weekly sessions; at least one principal investigator was present for all sessions.

Data were collected over a 10 -week period during the athletes' off season, following the protocol $|16-17|$. Pretesting was completed during the first week of the study and consisted of the Dynavision ${ }^{\mathrm{TM}}$ D2 Mode A and vision testing (identified above). Athletes completed four trials using Mode A; the first three were trial runs for familiarization, and the fourth run was recorded as the initial baseline score.

During weeks two and three, all athletes from both Groups A and B completed 15 minutes of Dynavision $^{\mathrm{TM}}$ D2 training, utilizing Modes A and B. Time length for Mode $B$ was calculated by subtracting 0.05 seconds from the final run of Mode A. These two weeks of training were an effort to familiarize all athletes with the Dynavision ${ }^{\mathrm{TM}} \mathrm{D} 2$ and to prevent a learning effect for those in Group A.

The next six weeks included group-specific intervention: 15 minutes, twice per week, following the established protocol ${ }^{|16-17|}$. Each week, Group A engaged in Dynavision ${ }^{\mathrm{TM}} \mathrm{D} 2$ training with Modes $\mathrm{A}, \mathrm{B}$, and $\mathrm{C}$ 
to address reaction time, peripheral field awareness, and central peripheral integration. Group B engaged in non-machine, therapist-led training to address similar skills. Intervention with Group B incorporated frequently used therapy items to mirror skills addressed using the Dynavision ${ }^{\mathrm{TM}} \mathrm{D} 2$ (see Table 1).

During the final week of the study, athletes completed post testing using the same assessments and technique as at pretest in week one. Follow-up testing at one- and two-months post intervention were completed to determine skill retention despite no further intervention.

\subsection{Data analysis}

Data analysis was completed using Statistical Package for Social Sciences (SPSS) Version 25. A Repeated Measures Analysis of Variance ( $A N O V A$ ) was used to analyze changes in means across multiple observations (pretest, posttest, and follow-up testing) withinand between-subject factors (Groups A and B) to determine differences among Dynavision ${ }^{\mathrm{TM}} \mathrm{D} 2$ quadrant and ring hits and speeds of reaction time. Parametric testing was used as data followed a normal distribution. Statistical significance was set at $\alpha \leqslant 0.05$.

\section{Results}

Twenty-two athletes participated in the study. Participants consisted primarily of freshmen $(n=10)$, followed by juniors $(n=5)$, seniors $(n=4)$, and sophomores $(n=3)$. During the study, one participant withdrew from the softball team, and her data were not included in the analysis. Of the 21 enrolled participants (Group
A $(n=10)$ and Group B $(n=11)$ ), all demonstrated typical results on vision testing items. Pretesting, post testing, and one-month and two-month follow-up testing were completed using the Dynavision ${ }^{\mathrm{TM}} \mathrm{D} 2$ Mode A to determine changes in quadrant and ring hits and speeds of reaction time for all participants.

2.1 Quadrant-specific results

2.1.1 Quadrant hits Baseline data for quadrant hits were similar for both groups for quadrants 1 (Group $\mathrm{A}: \mathrm{M}=18, \mathrm{SD}=4.11$, Group $\mathrm{B}: \mathrm{M}=18.7, \mathrm{SD}=4.13$ ) and 3 (Group A: $M=18.9, S D=2.96$, Group B: $M=19.7, S D=$ 3.41 ), but Group A displayed higher mean hits at baseline for quadrants 2 (Group A: $M=20, S D=2.63$, Group B: $M=17.6, S D=3.56$ ) and 4 (Group A: $M=20.4, S D=$ 2.68 , Group B: $M=18.5, S D=2.98$ ), though these differences were not statistically significant. Data followed normal distribution and did not violate sphericity. Overall effects of subjects within both Groups A and $\mathrm{B}$ demonstrated statistically significant improvements in mean hits in quadrant $1(\Lambda=0.48, F(3,57)$ $=4.15, P=0.01 ; \eta^{2}=0.18$ with observed power at 0.83$)$, quadrant $2\left(\Lambda=0.51, F(3,57)=4.75, P=0.005 ; \eta^{2}=0.20\right.$ with observed power at 0.88$)$, and quadrant $4(\Lambda=0.50$, $F(3,57)=5.67, P=0.002 ; \eta^{2}=0.23$ with observed power at 0.93 ).

Between group comparisons revealed a significant difference among quadrant $2(P=0.007)$ and quadrant $4(P=0.030)$, with Group A improving in hits more significantly than Group B. Significant quadrant hit data with between group comparisons are displayed in Table 2.

Table 2 Significant Between-Group Results for Quadrant Hits $(\bar{x} \pm s)$

\begin{tabular}{ccccccc}
\hline Item & Group & Pretest & Posttest & Follow-Up 1 & Follow-Up 2 & $P$ value \\
\hline \multirow{2}{*}{ Quadrant 2 } & Group A & $20.00 \pm 2.63$ & $24.20 \pm 5.18$ & $25.20 \pm 5.51$ & $24.10 \pm 4.70$ & 0.007 \\
& Group B & $17.55 \pm 3.56$ & $19.73 \pm 3.61$ & $21.45 \pm 4.18$ & $21.00 \pm 5.62$ & \\
\multirow{2}{*}{ Quadrant 4 } & Group A & $20.40 \pm 2.68$ & $23.50 \pm 4.65$ & $23.10 \pm 3.67$ & $25.00 \pm 4.95$ & 0.030 \\
& Group B & $18.45 \pm 2.98$ & $21.36 \pm 6.39$ & $19.00 \pm 3.44$ & $23.91 \pm 3.27$ & \\
\hline
\end{tabular}

2.1.2 Quadrant speed Pretest quadrant speeds, as measured by reaction time in seconds, were similar for both groups within all quadrants: Quadrant 1 (Group A: $M=0.80, S D=0.11$, Group B: $M=0.82, S D=0.06$ ), quadrant 2 (Group A: $M=0.77, S D=0.07$, Group B: $M=0.77, S D=0.13$ ), quadrant 3 (Group A: $M=0.77$, $S D=0.06$, Group B: $M=0.80, S D=0.06$ ), and quadrant 4 (Group A: $M=0.76, S D=0.07$, Group B: $M=0.76, S D=$ $0.10)$.

During analysis of quadrant speeds, data for quadrants 1, 3 and 4 did not violate sphericity; however, quadrant 2 data did violate sphericity. Thus, Greenhouse-Geisser correction was used for quadrant 2. With this correction, within-group data for both Groups $\mathrm{A}$ and $\mathrm{B}$ demonstrated statistically significant im- provements in reaction time within all quadrants: Quadrant $1\left(\Lambda=0.34, F(3,57)=19.25, P=0.000 ; \eta^{2}=0.50\right.$ with observed power at 1.00$)$, quadrant $2(\Lambda=0.36, F$ $(1.805,57)=10.64, P=0.000 ; \eta^{2}=0.36$ with observed power at 0.98$)$, quadrant $3(\Lambda=0.21, F(3,57)=20.04$, $P=0.000 ; \eta^{2}=0.51$ with observed power at 1.00$)$, and quadrant $4\left(\Lambda=0.38, F(3,57)=13.78, P=0.000 ; \eta^{2}=0.42\right.$ with observed power at 1.00$)$.

Between group comparisons revealed a significant difference among quadrant $1(P=0.002)$ and quadrant $2(P=0.006)$, with Group A improving in reaction time more significantly than Group B. Significant between group comparisons for quadrant speed data, as measured by reaction time in seconds, are displayed in Table 3. 
Table 3 Significant Between-Group Results for Quadrant Speeds $(\bar{x} \pm s)$

\begin{tabular}{ccccccc}
\hline Item & Group & Pretest & Posttest & Follow-Up 1 & Follow-Up 2 & $P$ value \\
\hline \multirow{2}{*}{ Quadrant 1 } & Group A & $0.80 \pm 0.11$ & $0.66 \pm 0.07$ & $0.63 \pm 0.05$ & $0.65 \pm 0.04$ & 0.002 \\
& Group B & $0.82 \pm 0.06$ & $0.75 \pm 0.08$ & $0.72 \pm 0.08$ & $0.80 \pm 0.09$ & $0.61 \pm 0.04$ \\
Quadrant 2 & Group A & $0.77 \pm 0.07$ & $0.63 \pm 0.08$ & $0.63 \pm 0.05$ & 0.05 & 0.006 \\
& Group B & $0.70 \pm 0.13$ & $0.77 \pm 0.10$ & $0.72 \pm 0.08$ & $0.71 \pm 0.08$ & \\
\hline
\end{tabular}

\subsection{Ring-specific Results}

2.2.1 Ring hits Baseline data for ring hits were similar for both groups for rings 1, 2, 4 and 5: Ring 1 (Group A: $M=9.20, S D=4.19$, Group B: $M=9.27, S D=2.53)$, ring 2 (Group A: $M=10.80, S D=3.43$, Group B: $M=11.18$, $S D=2.64$ ), ring 4 (Group A: $M=18.00, S D=2.45$, Group B: $M=18.00, S D=4.54$ ), and ring 5 (Group A: $M=18.80$, $S D=4.37$, Group B: $M=17.55, S D=3.59)$. Within ring 3, Group A baseline $(M=20.40, S D=4.77)$ was slightly higher than Group B $(M=18.45, S D=3.53)$. Data followed normal distribution and did not violate sphericity.
Overall effects of subjects within both Groups A and $\mathrm{B}$ demonstrated statistically significant improvements in mean hits in ring $3(\Lambda=0.67, F(3,57)=3.29$, $P=0.03 ; \eta^{2}=0.15$ with observed power at 0.72$)$, ring 4 $\left(\Lambda=0.43, F(3,57)=6.14, P=0.001 ; \eta^{2}=0.24\right.$ with observed power at 0.95$)$, and ring $5(\Lambda=0.32, F(3,57)=$ 9.22, $P=0.000 ; \eta^{2}=0.33$ with observed power at 1.00 ).

Between group comparisons revealed a significant difference among ring $2(P=0.033)$ and ring $3(P=$ 0.014 ), with Group A improving in hits more significantly than Group B. Significant ring hit data with between group comparisons are displayed in Table 4.

Table 4 Significant Between-Group Results for Ring Hits $(\bar{x} \pm s)$

\begin{tabular}{ccccccc}
\hline Item & Group & Pretest & Posttest & Follow-Up 1 & Follow-Up 2 & $P$ value \\
\hline \multirow{2}{*}{ Ring 2 } & Group A & $10.80 \pm 3.43$ & $13.80 \pm 2.49$ & $10.00 \pm 2.63$ & $12.20 \pm 3.43$ & 0.033 \\
& Group B & $11.18 \pm 2.64$ & $10.36 \pm 2.66$ & $10.36 \pm 3.91$ & $8.82 \pm 3.10$ & \\
\multirow{2}{*}{ Ring 3 } & Group A & $20.40 \pm 4.77$ & $25.60 \pm 5.17$ & $25.30 \pm 4.52$ & $21.90 \pm 4.23$ & 0.014 \\
& Group B & $18.45 \pm 3.53$ & $18.91 \pm 3.42$ & $22.00 \pm 5.87$ & $20.18 \pm 6.54$ & \\
\hline
\end{tabular}

2.2.2 Ring speed Ring speeds were a measure of reaction time (in seconds) from central and peripheral regions of the light board. Pretest scores were similar for both groups within rings 1, 2, and 3: Ring 1 (Group A: $M=0.63, S D=0.06$, Group B: $M=0.64, S D=0.06)$, ring 2 (Group A: $M=0.68, S D=0.05$, Group B: $M=0.69$, $S D=0.05$ ), and ring 3 (Group $\mathrm{A}: M=0.72, S D=0.07$, Group B: $M=0.72, S D=0.06$ ). Within rings 4 and 5, however, Group A demonstrated a slightly faster baseline: Ring 4 (Group A: $M=0.81, S D=0.07$, Group B: $M=0.88, S D=0.07$ ), and ring 5 (Group A: $M=0.92, S D=$ 0.09 , Group B: $M=0.97, S D=0.08$ ).

During data analysis of ring speeds, data for rings 1 , 3, 4 and 5 did not violate sphericity; however, ring 2 data did violate sphericity. Thus, Greenhouse-Geisser correction was used. With this correction, withingroup data for both Groups A and B demonstrated sta- tistically significant improvements in reaction time for all rings: Ring $1\left(\Lambda=0.12, F(3,57)=53.30, P=0.000 ; \eta^{2}=\right.$ 0.74 with observed power at 1.00$)$, ring $2(\Lambda=0.18, F$ $(2.33,57)=41.43, P=0.000 ; \eta^{2}=0.69$ with observed power at 1.00$)$, ring $3(\Lambda=0.16, F(3,57)=29.59, P=0.000$; $\eta^{2}=0.61$ with observed power at 1.00$)$, ring $4(\Lambda=0.12$, $F(3,57)=36.45, P=0.000 ; \eta^{2}=0.66$ with observed power at 1.00$)$, and ring $5(\Lambda=0.30, F(3,57)=14.00, P=$ $0.000 ; \eta^{2}=0.42$ with observed power at 1.00 ).

Between group comparisons revealed a significant difference among ring $2(P=0.002)$, ring $4(P=0.003)$, and ring $5(P=0.004)$, with Group $A$ improving in reaction time more significantly than Group B. Significant results between groups for ring speed data, as measured by reaction time in seconds, are displayed in Table 5 .

Table 5 Significant Between-Group Results for Ring Speeds $(\bar{x} \pm s)$

\begin{tabular}{|c|c|c|c|c|c|c|}
\hline Item & Group & Pretest & Posttest & Follow-Up 1 & Follow-Up 2 & $P$ value \\
\hline \multirow[b]{2}{*}{ Ring 2} & Group A & $0.68 \pm 0.05$ & $0.53 \pm 0.07$ & $0.53 \pm 0.04$ & $0.52 \pm 0.03$ & \multirow[b]{2}{*}{0.002} \\
\hline & Group B & $0.69 \pm 0.05$ & $0.60 \pm 0.06$ & $0.60 \pm 0.04$ & $0.58 \pm 0.03$ & \\
\hline \multirow{2}{*}{ Ring 4} & Group A & $0.81 \pm 0.07$ & $0.66 \pm 0.07$ & $0.68 \pm 0.07$ & $0.67 \pm 0.06$ & \multirow{2}{*}{0.003} \\
\hline & Group B & $0.88 \pm 0.07$ & $0.79 \pm 0.08$ & $0.75 \pm 0.08$ & $0.78 \pm 0.09$ & \\
\hline \multirow[b]{2}{*}{ Ring 5} & Group A & $0.92 \pm 0.09$ & $0.81 \pm 0.08$ & $0.78 \pm 0.10$ & $0.77 \pm 0.08$ & \multirow[b]{2}{*}{0.004} \\
\hline & Group B & $0.97 \pm 0.08$ & $0.90 \pm 0.10$ & $0.88 \pm 0.07$ & $0.89 \pm 0.07$ & \\
\hline
\end{tabular}




\section{Discussion}

This study investigated the effectiveness of the Dynavision ${ }^{\mathrm{TM}} \mathrm{D} 2$ in comparison to non-machine, therapist-led vision training in improving peripheral field awareness among collegiate softball athletes. Previous research incorporating the Dynavision ${ }^{\mathrm{TM}} \mathrm{D} 2$ for outcomes of enhanced sport performance among collegiate athletes have focused primarily on hits and reaction time [11]. While it may be possible to improve peripheral field awareness through visual training [34], studies have not been replicated with a collegiate athlete population. Still, much of the research in this area focuses on reaction time, with little emphasis on peripheral visual field training.

Results revealed that both machine and therapist-led visual training can improve peripheral field awareness. Results of a number of lights hit indicated similarities among Groups A and B, with each group demonstrating improvements. However, analysis of these findings demonstrated that, overall, Group A had more significant gains in a number of quadrant hits. Significance was noted within quadrants 2 and 4 between groups, with Group A demonstrating a more significant improvement in a number of quadrant hits. While no other studies compared two groups and included analysis by quadrant, authors anticipate this increase in hits in quadrants 2 and 4 is due to these quadrants being located on the right half of the board. Since the majority of athletes in this study were right eye dominant and right handed, operating in the right visual field may have been more instinctive and easier to train.

Analysis of quadrant speed data is in alignment with previous research on reaction time ${ }^{[11]}$. These findings indicated a significant reduction in reaction for both groups within each quadrant, except for Group B in quadrant 2 . Statistical significance was found between groups for quadrants 1 and 2. Overall, Group A demonstrated a greater reduction in speed as well as better maintenance at follow-up. Regarding reaction time for each of the five concentric rings, significant differences between groups for rings 2, 4, and 5 may relate to preference for the right and upper visual field, although number data within each ring and quadrant were not cross analyzed. Athlete stance may have also contributed to the ability to visualize more of the board, especially for outer rings, using peripheral vision instead of head turns. Rings furthest from the central field $(3,4$, and 5$)$ require more peripheral awareness and were found to have improved reaction time within Groups A and B. These findings are similar to findings from Clark and colleagues (2015) ${ }^{[9]}$, noting that reaction time in ring 5 (that furthest from the central field) was indicative of the slowest reaction time.

Similarities in reaction time and retention suggest that both the Dynavision ${ }^{\mathrm{TM}} \mathrm{D} 2$ and non-machine, therapist-led visual training methods may be effective interventions for enhancing visual field awareness of an athletic population. However, the Dynavision ${ }^{\mathrm{TM}} \mathrm{D} 2$ was more effective in reducing reaction time, improving peripheral awareness (quadrant and rings), and improving number of hits across intervention and follow-up. Retention testing revealed that Group A retained skills better than Group B. This may be associated with a learning effect due to Group $\mathrm{A}$ using the Dynavision ${ }^{\mathrm{TM}} \mathrm{D} 2$ in more sessions than Group B.

Additional research in this area will strengthen findings and promote the role of occupational therapists as interventionists in such studies. Occupational therapists working directly with a sports team, consulting with an optometrist or other eye care professional, or aiming to extend expertise in vision outside of traditional practice may consider incorporating the Dynavision $^{\mathrm{TM}} \mathrm{D} 2$ into interventions with an athlete population. Despite reimbursement for such services shifting from traditional methods of payment, potential exists to serve a new clientele and to assist in advancing meaningful participation in sports. Providing services to a well population is an expanding area of health promotion within occupational therapy, one with which the intervention described in this study aligns. Utilizing motivation, an occupational therapist incorporates physical, cognitive, and sensory challenges as methods to contribute to improvements in athletic performance.

\section{Limitations}

Despite Group A receiving more sessions with the Dynavision $^{\mathrm{TM}} \mathrm{D} 2$, this limitation was minimized by allowing a two-week familiarization period in which both groups had the same exposure. The Dynavision ${ }^{\mathrm{TM}}$ D2 was used for both training as testing. While this allows for versatility of the device, it may also allow for athletes to become familiar with anticipating testing processes. Despite this, the location and sequence of lights remained random, causing novel reactions to un-anticipated stimuli.

\section{Conclusion}

Peripheral vision contributes to sport performance, with implications on an athlete's reaction time, anticipation, and overall safety while on the field ${ }^{[3,4,9]}$. Research indicates improvement of visual field awareness can be gained through use of the Dynavision ${ }^{\mathrm{TM}}$ D2 ${ }^{[18-19]}$, yet these studies primarily involve subjects with established field loss or post injury ${ }^{[20]}$, rather than as a training method among well athletes. The results from this study demonstrate the adaptability of the visual system for those with normal field of vision. By addressing reaction time, peripheral awareness, and central-peripheral integration following a consistent protocol, in this case that of ${ }^{[16-17]}$, improvements were made, and maintenance of skills was demonstrated. This indicates that visual training using Dynavision ${ }^{\mathrm{TM}}$ D2 and non-machine strategies are effective methods in increasing the visual field awareness of a collegiate athlete population, yet findings from this research 
should be replicated and applied to additional populations.

\section{References}

[1] American Optometric Association. Important visual skills for sports [EB/OL]. (2018-11-10) [2019-08-15]. https://www.aoa. org/patients - and-public/caring - for-your-vision/sports - and - vision/important-vision-skills-for-sports.

[2] KNUDSON D, KLUKA D A. The impact of vision and vision training on sport performance [J]. JOPERD, 1997, 68(4): 17-24.

[3] SCHWAB S, MEMMERT D. The impact of a sports vision training program in youth field hockey players $[\mathrm{J}]$. J Sports Sci Med, 2012, 11(4): 624-631.

[4] WILLIAMS A M, DAVIDS K. Assessing cue usage in performance contexts: A comparison between eye movement and verbal report methods $[\mathbf{J}]$. Behavioral Research Methods, Instruments, and Computers, 1997, 29(3): 364-375.

[5] THOMAS C, ANDRICH P, ABRAMS-MOTZ P. Comparison of three types of vision therapy exercises on visual skills of sport performance $[\mathrm{J}]$. Optom Vis Performance, 2017, 5(1): 21-26.

[6] MADSEN B, BLAIR K. Oregon State University Softball: Dynamic visual acuity training for improving performance $[\mathrm{J} / \mathrm{OL}]$. SportRxiv Preprints, (2017-08-19) [2019-08-16]. https://doi.org/10.31236/ osf.io/nwqpj.

[7] ERICKSON G B, ERICKSON O D. Optimizing visual performance for sport $[\mathrm{J}]$. Adv Ophthalmol Optom, 2018, 3(1): 1-19.

[8] APPELBAUM L G, LU Y, KHANNA R, et al. The effects of sports vision training on sensorimotor abilities in collegiate softball athletes [J]. Athletic Training \& Sports Health Care, 2016, 8(4): 154163.

[9] CLARK J F, GRAMAN P, ELLIS J K, et al. An exploratory study of the potential effects of vision training on concussion incidence in football $[\mathrm{J}]$. Optometry \& Visual Performance, 2015, 3(2): 116125 .

[10] Dynavision International LLC. Dynavision: About [EB/OL]. (2018-01-12) [2019-08-10]. https://www.dynavisioninternational.com/about.

[11] CROSS A K, BROCKEVELT B L, KRUISSELBRINK A R, et al.
The effectiveness of Dynavision training to improve visual motor skills of collegiate athletes: A pilot study $[\mathrm{J}]$. Gazzetta Medica Italiana-Archivo Per Le Scence Medicine, 2013, 172 (7-8): 627634.

[12] WELLS A J, HOFFMAN J R, BEYER K S, et al. Reliability of the Dynavision ${ }^{\mathrm{TM}} \mathrm{d} 2$ for assessing reaction time performance $[\mathrm{J}]$. J Sports Sci Med, 2014, 13(1): 145-150.

[13] ANDERSON L, CROSS A, WYNTHEIN D, et al. Effects of dynavision training as a preparatory intervention status post cerebrovascular accident: a case report $[\mathrm{J}]$. Occup Ther Health Care, $2011,25(4): 270-282$

[14] GOLISZ K. Occupational therapy interventions to improve driving performance in older adults: a systematic review $[\mathrm{J}]$. Am J Occup Ther, 2014, 68(6): 662-669.

[15] HUNT L A, ARBESMAN M. 0. Evidence-based and occupational perspective of effective interventions for older clients that remediate or support driving performance $[\mathrm{J}]$. Am J Occup Ther, 2008, 62(2): 136-148.

[16] FELDHACKER D R. LUCAS MOLITOR W. Pilot study of an occupational therapy Dynavision D2 protocol for enhancement of visual performance among collegiate athletes $[\mathrm{J}]$. Annals of International Occupational Therapy, 2019, 2(2): 69-78.

[17] FELDHACKER D R, LUCAS MOLITOR W, ATHMANN A, et al. Efficacy of high-performance vision training on improving the reaction time of collegiate softball athletes: A randomized trial $[\mathrm{J}]$. Journal of Sports Medicine and Allied Health Science, 2019, 4(6): 1-12.

[18] GILLEN G, NILSEN D M, ATTRIDGE J,et al. Effectiveness of interventions to improve occupational performance of people with cognitive impairments after stroke: An evidence-based review [J]. Am J Occup Ther, 2015, 69(1): 6901180040p1-9.

[19] PAVLOVICH D. Optimal interventions for treatment of visual defects in individuals with an acquired brain injury $[\mathrm{J}]$. Occup Ther Comm, 2015, 15: 1-38.

[20] CLARK J F, ELLIS J K, BURNS T M, et al. Analysis of central and peripheral vision reaction times in patients with postconcussion visual dysfunction [J]. Clin J Sport Med, 2017, 27(5): 457461 .

\title{
改善大学垒球运动员视野意识的随机试验研究
}

\author{
Diana R. Feldhacker ${ }^{1}$, Whitney Lucas Molitor ${ }^{2}$ \\ 1 克瑞顿大学药学和卫生专业学院, 内布拉斯加州 奥马哈 68178 , 美国; \\ 2 南达科他州大学健康科学学院, 南达科他州 弗米利恩 57069 , 美国
}

\begin{abstract}
摘要 目的: 在大学垒球运动员中确定高效视觉训练对提高视野意识的作用, 并比较不同千预结果的有效 性。方法: 将 21 名运动员随机分为 2 组, A 组参加 Dynavision ${ }^{\mathrm{TM}}$ D2 训练, B 组参加治疗师指导非机械的视 觉训绕。按照预先建立的视觉训练方案, 2 组均 $15 \mathrm{~min}$ 进行 1 次视觉训练, 每周 2 次, 持续 6 周。经过预测 试后和 2 周的熟悉期, 2 组均可以使用Dynavision ${ }^{\mathrm{TM}} \mathrm{D} 2$ 训练设备。特定的小组千预措施包括认认知和感觉 方法为主的动态技能培训。采用重复测量方差分析 (ANOVA) 分析 2 组组内和组间在多个观察值(测前、测后 和后续测试) 中均值的变化, 以确定 2 组 Dynavision ${ }^{\mathrm{TM}} \mathrm{D} 2$ 象限、环命中数和反应速度的差异。参数测试用于 埒循正态分布的数据, 统计显著性设为 $\alpha \leqslant 0.05$ 。结果: 通过 Dynavision ${ }^{\mathrm{TM}} \mathrm{D} 2$ 象限和环内的平均命中率和速 度可以证明, 高效视觉训练能够有效提高大学垒球运动员的外周视野感知。A 组和 B 组在象限 1 、2 和 4 以 及第 3、4 和 5 环内命中数均具有统计学上的显著改善, 而且 2 组在所有象限和所有环内的速度均具有统计 学上的显著改善。因此, Dynavision ${ }^{\mathrm{TM}} \mathrm{D} 2$ 训练和替代性视觉训绕均能提高优秀运动人群的视野感知和反应 时间, 而 Dynavision ${ }^{\mathrm{TM}} \mathrm{D} 2$ 训练可能是更有效的方法。A 组象限 $2 、 4$ 和环 $2 、 3$ 的命中数以及象限 1 、2 和环 2 、 3 的速度均比 $\mathrm{B}$ 组在统计学上有显著差异。随访测试在没有额外干预的情况下完成, 并在 $1 、 2$ 个月的随访中 确认了研究结果和技能保留。结论: 外周视野有助于运动表现, 影响运动员在比赛中的反应时间、预见和整 体安全性。通过 Dynavision ${ }^{\mathrm{TM}} \mathrm{D} 2$ 训练可以提高视野感知, 以往研究主要涉及已确定视野变失或受伤后的受 试者而不针对训练运动员, 本研究结果证明其具有正常视野人群视觉系统的适应性。
\end{abstract}

关键词 Dynavision ${ }^{\mathrm{TM}} \mathrm{D} 2$ 动视仪;视觉训䋆; 作业治疗; 垒球;运动员; 视野; 外周视野

DOI : $10.3724 /$ SP.J.1329.2020.01007 